\title{
Cengestao
}

\section{O uso de Realidade Aumentada no contexto dos museus: o portfólio brasileiro de teses e dissertações até 2017}

\author{
Graciela Sardo Menezes \\ Mestre, Universidade Federal de Santa Catarina, Florianópolis, SC, Brasil; \\ graegra@gmail.com \\ William Barbosa Vianna \\ Doutor, Universidade Federal de Santa Catarina, Florianópolis, SC, Brasil; \\ wpwilliam@hotmail.com \\ Márcio Matias \\ Doutor, Universidade Federal de Santa Catarina, Florianópolis, SC, Brasil; \\ matias.m@ufsc.br
}

\begin{abstract}
Resumo: O objetivo deste estudo é identificar e analisar, dentro da literatura oriunda dos Programas de Pós-Graduação do Brasil, teses e dissertações que investigaram o uso das tecnologias digitais, nomeadamente a Realidade Aumentada, nos espaços museológicos. Busca subsidiar o desenvolvimento do tema com base em estudos consolidados nos Programas de Pós-Graduação brasileiros e identificar a fundamentação teórica para investigações futuras. Para tanto, foi realizado uso de busca simples, preliminar e de natureza exploratória nos Bancos de Teses e Dissertações da Coordenação de Aperfeiçoamento de Pessoal de Nível Superior e do Instituto Brasileiro de Informação em Ciência e Tecnologia. Os resultados apontaram para uma produção dispersa, pouco sistematizada e para a oportunidade de criação de protocolos de pesquisa no assunto com base nos dados levantados.
\end{abstract}

Palavras-chave: Museu. Realidade Aumentada. Ciência da Informação. Interdisciplinaridade. Museologia.

\section{Introdução}

No atual contexto, os usuários dos diversos equipamentos culturais familiarizados com as formas de interação disponibilizadas pelos ambientes digitais e estruturas cognitivas associadas - vêm demandando artefatos 
tecnológicos que suportem maior interação, participação ativa e autonomia a fim de obter experiências interativas mais ricas.

As características dos usuários conhecidos como "nativos digitais" (PRENSKY, 2001) indicam a necessidade de se buscar caminhos que conversem com seus modelos mentais, hábitos e interesses. Ao acompanhar a evolução do contexto informacional, os museus passaram a valorizar não só a divulgação online de suas atividades, mas também a forma e as ferramentas utilizadas no espaço expositivo.

Cury (2005) observa que a compreensão contemporânea do que é comunicação define que o público assume uma posição de maior poder, tornando-se sujeito ativo no processo comunicacional. A autora destaca ainda que os principais meios que os museus têm para se comunicar com seus públicos são as exposições museológicas e ações educativas. A forma como as informações são apresentadas aos usuários varia de acordo com a mídia utilizada e essa, por sua vez, de acordo com a tecnologia disponível.

As ferramentas de tecnologia digital, por sua vez, podem ampliar a participação dos usuários dos museus, ou mesmo fornecer informação que não seria viável disponibilizar de forma ampliada via web. Observa-se a tendência de expandir o acesso na criação de museus virtuais, utilização de recursos multimídia, audiovisuais e nos dispositivos interativos comandados pelo visitante que conversam com o ambiente em tempo real.

Neste contexto, a Realidade Aumentada (RA) pode ser compreendida como aliada no processo dialógico do público para com ambientes e artefatos culturais e museológicos. Compreendida como uma ferramenta capaz de fornecer informações que se mesclam com o entorno, a RA é observada no cenário cultural desde o surgimento desse recurso, aparecendo em simulações imagéticas em 3D ou na disponibilização de descrições e informações sobre obras ou objetos, além das tradicionalmente proporcionadas.

Em geral, os estudos relacionados ao uso de RA têm se concentrado na área de Ciência da Computação e Design de Interação e nas engenharias. No campo da CI (Ciência da Informação), existem pesquisas que investigam a 
utilidade desta ferramenta dentro das Bibliotecas e Arquivos (ZAGATTO, 2013).

Ao mesmo tempo, parece interessante identificar quais estudos abordam a RA nos espaços museológicos no campo da Ciência da Informação no Brasil, notadamente teses e dissertações nos Programas de Pós-Graduação da área, considerando tal produção intelectual como fundamento de futuros estudos sobre o assunto na área da Ciência da Informação. Parte-se do pressuposto de que podem haver lacunas na literatura e oportunidades de investigação sobre o uso de RA no contexto dos espaços de exposição dos museus, com investigação específica no campo da CI.

Tendo em vista o exposto, parte-se da seguinte questão de pesquisa: que teses e dissertações no contexto brasileiro da CI têm investigado sobre o uso da RA nos espaços museológicos?

O objetivo deste estudo é identificar e analisar, dentro da literatura oriunda dos Programas de Pós-Graduação do Brasil, teses e dissertações que investigaram o uso das tecnologias digitais, nomeadamente a RA, nos espaços museológicos.

Justifica-se em vista do desenvolvimento do tema na CI, uma vez que, com base nos resultados, será possível identificar a representatividade dos Programas em Ciências da Informação na abordagem desse assunto e, eventualmente, proporcionar uma fundamentação teórica para investigação futura que pretende abordar a Experiência dos Usuários na interação com RA no Museu de Arqueologia e Etnologia (MarquE) da Universidade Federal de Santa Catarina (UFSC).

Para tanto, buscou-se identificar possíveis lacunas e contribuições, bem como os autores que vêm tratando o assunto no âmbito dos Programas de PósGraduação estudados. O presente trabalho objetivou ser uma investigação preliminar de natureza predominantemente quantitativa e exploratória. $\mathrm{O}$ método utilizado foi uma busca simples nos Bancos de Teses e Dissertações da Coordenação de Aperfeiçoamento de Pessoal de Nível Superior (CAPES) e Instituto Brasileiro de Informação em Ciência e Tecnologia (IBICT). 
Foi realizada uma seleção inicial nos referidos repositórios por meio da leitura dos títulos. O critério de escolha priorizou os trabalhos que tratam as tecnologias digitais no contexto das recepções em museus.

A fim de ampliar o leque de artigos recuperados, o segundo critério utilizado foi o de selecionar os que tratam do uso de tecnologias digitais interativas nos museus. Com base nos resumos, foram selecionadas as publicações alinhadas com os objetivos da proposta de estudo. Para a extração dos dados e análise utilizou-se um checklist, apresentado no tópico "Procedimentos Metodológicos".

\section{Desafios para o avanço da Interdisciplinaridade na Ciência da Informação}

Segundo Almeida (2016), bibliotecas, museus e arquivos são instituições que nunca chegaram a ter suas fronteiras bem demarcadas. A formação, tanto do museu antigo quanto da biblioteca, registrava a preocupação de reunir e organizar coleções para preservá-las.

Há quase três décadas, Homulus (1990) já afirmava que museus, arquivos e bibliotecas têm muitas características em comum e fazem parte de um espectro ou continuum de instituições que possuem necessidades específicas, com problemas comuns e diferenças entre essas instituições que tenderiam a desaparecer com a era eletrônica.

Smit (2017), por sua vez, considera que a CI representa uma área do conhecimento que agrega valor às soluções práticas para diversos tipos de unidade de informação, desdobrando-se em diferentes contextos científicos de aplicações institucionais e profissionais, tipos de espaços de informação (Arquivos, Bibliotecas e Museus) e memória (acervos e serviços) que são denominados pelo codinome "3 Marias" (SMIT, 1993, p. 81).

Considera-se, para fins deste estudo, que a distinção entre os diferentes contextos, baseada nos tipos de documentos custodiados, foi ultrapassada há 
tempos e teve seu fim decretado com o advento do documento digital, presente em todos os espaços de forma cada vez mais incontornável (SMIT, 2017).

Nesse sentido, esse estudo embasa-se também no reconhecimento de que o campo da CI, de maneira geral, utiliza-se da tecnologia da informação (SARACEVIC, 1996; SHERA; CLEVELAND, 1977). Os avanços tecnológicos obtidos nos últimos anos impulsionaram o modo como as informações são tratadas, organizadas, compartilhadas e utilizadas. Os recursos digitais estão alterando constantemente a forma como as pessoas relacionam-se com a informação.

A conexão em rede, os computadores pessoais, os smartphones e tablets já fazem parte do cotidiano de grande parte da população mundial. Com isso, hábitos e modelos de relacionamentos estão sendo criados e adaptados às novas e constantes possibilidades de interação, sendo que na presente proposta o direcionamento orienta-se para as questões relacionadas à interação dos usuários com tecnologias digitais nos espaços museológicos.

A personalização da forma como o usuário busca e recupera a informação descreve seu comportamento informacional, que depende de uma gama de variáveis tanto relacionadas aos indivíduos quanto ao meio e mídia empregados; e a adaptação dos museus às demandas de seus públicos possibilitou a abertura de novos espaços expositivos.

Muchacho (2005) ressalta que as novas tecnologias apoiam as instituições, tornando-as mais atrativas por meio de múltiplos recursos que oferecem outras experiências sensoriais e cognitivas. Um dos conceitos inovadores proposto pelas tecnologias digitais é possibilitar a interação entre o utilizador e os conteúdos expostos, estabelecendo novas regras da interação entre os museus e os seus públicos (SOUSA, 2007).

Sendo uma ciência interdisciplinar, considera-se cientificamente válido que a CI dialogue com as demais ciências que abordam temas sensíveis às práticas compartilhadas, como é o caso da RA, particularmente frente ao ponto de inflexão que é a realidade digital. 
Frequentemente, abordagens que priorizam os princípios de um único campo de conhecimento falham ao não considerar de modo mais abrangente os elementos que as circundam. A abertura para o diálogo com especialistas de outras áreas do conhecimento é uma característica da CI desde sua fundação, e pode ser considerada uma possibilidade epistemológica nova (LEMOS, 2016).

Segundoa especificidade da CI não é o estudo de um novo objeto empírico, mas a análise dos mesmos fenômenos já estudados por outras ciências, a partir do ponto de vista diferente - o olhar informacional (ARAÚJO, 2014, p. 8).

Portanto, as investigações que visam compreender e articular as diversas disciplinas que envolvem um problema complexo de informação contribuem para consolidar a atuação da CI no âmbito acadêmico, científico e tecnológico; envolvem processos como recepção e mediação em museus e emprego de tecnologias de RA sob o enfoque da informação.

Estudos realizados por Zagatto (2013) revelam que as publicações no Journal of American Society for Information Science and Technology (JASIST, online) sobre esta temática superam em quantidade os 10 periódicos brasileiros da área da CI utilizados na comparação. O primeiro apresentou um total de 16 artigos, enquanto o periódico brasileiro "Informação \& Sociedade: estudos" foi o que apresentou mais resultados dentre os 10 investigados, totalizando 7 artigos. Uma rápida busca na Base de Dados Referenciais de Artigos de Periódicos em Ciência da Informação (BRAPCI), em novembro de 2017, utilizando a seguinte lógica de busca: todos os campos $\{$ "RA" $\}$, recuperou um total de apenas 6 títulos, corroborando a pertinência do desenvolvimento de pesquisas como o da presente proposta.

\subsection{Recepção em museus e as tecnologias digitais}

Museus são espaços de memória a serviço da sociedade que têm por finalidade adquirir, conservar, pesquisar, comunicar e transmitir o patrimônio material e imaterial da humanidade e seu ambiente para fins de estudo, educação e lazer 
(MUSEUM..., 2007). As exposições constituem a principal forma de comunicação na qual o visitante tem contato com os objetos museais.

Desvallées e Mairesse (2013) destacam o caráter unilateral do modelo comunicacional aplicado nos museus, denunciando um aspecto linear e hierárquico de comunicação. Tal percepção demonstra o pensamento e as práticas predominantes em um tempo não muito distante. Atualmente, o uso das Tecnologias da Informação e Comunicação (TIC), à serviço de unidades de informação (bibliotecas, arquivos, museus), tem potencial para facilitar e tornar os processos e serviços interativos, sejam eles internos ou externos.

A aplicação de ferramentas da tecnologia digital tornou possível a prática de interações bilaterais, em processos dinâmicos nos quais os interlocutores constroem, em conjunto, significados e conceitos. A comunicação museológica só é efetivada quando o visitante incorpora o discurso expositivo, o integra a seu cotidiano, (re) elabora-o na forma de um novo discurso do qual o museu se apropria, adapta e difunde novamente (CURY, 2005).

A seguir, é apresentada uma seleção com alguns recursos tecnológicos identificados na literatura e utilizados por museus em seu processo de comunicação com seus públicos externos (visitantes e pesquisadores).

a) quiosques de informação - São móveis ou aparatos interativos que disponibilizam informações aos visitantes por meio de telas de computador, interativas ou não (CHELINI, 2012). Para Veloso (2015), quiosques de informação também podem ser conhecidos como Totens Digitais. A informação é apresentada por meio de uma interface digital na qual o usuário navega por links e hipertextos ou assiste a vídeos e animações de forma passiva. Os temas disponibilizados podem estar relacionados às exposições, à própria instituição (mapas do local, histórico, missão etc.) ou servir de recurso complementar ao percurso expositivo. Um exemplo deste diálogo são os totens do Museu da Língua Portuguesa $^{1}$, que ofereciam ${ }^{2}$ quizes interativos aos visitantes como forma de entretenimento e apêndice dos tópicos expostos; 


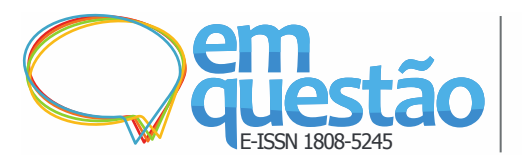

O uso de Realidade Aumentada no contexto dos museus: o portfólio brasileiro de teses e dissertações até 2017

Graciela Sardo Menezes, William Barbosa Vianna e Márcio Matias

b) tours virtuais - Considerados, na presente proposta, análogos aos Museus Virtuais. São ambientes digitais que simulam ambientes reais (idênticos à instituição ou não) e oferecem experiências de interatividade (CARVALHO, 2008). Podem aparecer como representação de toda a instituição, de parte de uma exposição ou estar relacionada apenas a algum objeto específico. Tal recurso oferece a possibilidade de explorar informações intangíveis, do ponto de vista material, por meio de simulações. Exemplos são as visitas virtuais oferecidas pela Casa Portinari $^{3}$ e pelo projeto Google Arts \& Culture ${ }^{4}$;

c) QR-Code (Quick Response Code ${ }^{5}$ ) - Um desdobramento do Código de Barras. São basicamente símbolos bidimensionais simplificados que podem ser lidos de maneira rápida. Inicialmente, seu diferencial destacase pela capacidade de ser lido por câmeras de celulares, smartphones e tablets, o que contribuiu para sua ampla difusão e uso (LIU; YANG; LIU, 2008; REIS; BARBOSA, 2014). O programador sênior Ronaldo Prass esclarece que após a decodificação, por meio de um aplicativo instalado no dispositivo móvel, esse código passa a ser um trecho de texto e/ou um link que irá redirecionar o acesso ao conteúdo publicado em algum site (PRASS, 2011). Também é considerado um caso de RA por disponibilizar acesso a outros recursos multimídia quando da interação com objetos reais. Em museus pode ser um aliado no fornecimento de informações sobre obras, exposições e instituições de maneira interativa e dinâmica. Por se tratar de acesso via dispositivo móvel, favorece a utilização em espaços abertos, monumentos históricos e sítios arqueológicos. O Museu Dr. Joaquim Manso ${ }^{6}$ utiliza este recurso para fornecer mais informações sobre as obras expostas em suas galerias;

O Museu Etnográfico e Arqueológico do Dr. Joaquim Manso, também conhecido por Museu da Nazaré - Portugal, abriu ao público em 1976. Encontra-se instalado em uma moradia do princípio do séc. XX, a antiga casa de veraneio do Dr. Joaquim Manso, escritor e jornalista, fundador do "Diário de Lisboa”. Esta casa foi doada ao Estado em 1968 pelo benemérito nazareno Amadeu Gaudêncio para nela ser instalado o museu. Sendo um museu de 
carácter regional, tem um acervo que documenta a história da região e a identidade cultural do povo de Nazaré. Recentemente foi reforçada a vocação do museu no domínio da etnografia marítima, dando relevo às atividades piscatórias com a exposição de diferentes tipos de embarcações tradicionais e artes da pesca. Fazem também parte da coleção achados arqueológicos, registos e trajes tradicionais, assim como algumas peças de escultura e pintura ${ }^{7}$.

d) beacon - é uma tecnologia baseada no conceito "internet das coisas" que utiliza processos BLE (Bluetooth Low Energy). Trata-se de um sistema de posicionamento que emite sinais capazes de serem detectados por dispositivos móveis tais como tablets e smatphones que tenham o aplicativo (TagPoint) instalado. Em museus, na medida em que o visitante se desloca pela exposição, ao aproximar-se da zona de transmissão - vinculada a um espaço físico, objeto, monumento etc. um aviso é enviado automaticamente e dados sobre as obras são disponibilizados. Além disso, esta tecnologia é capaz de encontrar os interesses dos visitantes, ou potencial interesse, a partir de seus comportamentos, fornecendo informações direcionadas a cada usuário próximo aos pontos de acesso (HE; CUI; ZHOU, 2015; APLICATIVO..., 2016). Semelhantemente ao QR-Code, esta tecnologia permite que os visitantes obtenham mais informações e detalhes sobre os objetos vinculados ao sinal, facilitando o processo de obtenção de conhecimento nos museus. Com praticamente os mesmos recursos e funções, a ferramenta RFID (radio frequency identification identificação por radiofrequência) difere no tipo de sinal que suporta, uma vez que utiliza frequências de rádio.

Essas são algumas das possibilidades que as tecnologias digitais apresentam no que tange aos processos de comunicação em espaços museológicos. Longe de esgotar o leque de opções, os itens listados fornecem uma base para compreender o panorama geral e as vantagens que oferecem. Contudo, sua utilização não é compulsória ou necessária. Em muitos casos, tais aparatos digitais não dialogam com a proposta institucional do museu. Cabe a 
cada instituição verificar a coerência e pertinência de sua aplicação para evitar o uso indiscriminado e fora de contexto.

Para Pinho (2007), as visitas presenciais são insubstituíveis na medida em que a experiência estética do contato direto com o bem cultural não se pode reproduzir, nem num ambiente digital.

A RA pode utilizar alguns desses processos para amparar seus sistemas complexos. A seguir, é apresentado o aprofundamento sobre este conceito.

\subsection{A Realidade Aumentada (RA)}

Com a ampla utilização de dispositivos móveis em diversas tarefas do cotidiano, a RA ganha destaque, sendo possível perceber que o interesse pelo tema é crescente e o público se mostra receptivo à sua utilização (SILVA, 2015). O uso de RA permite ao usuário interagir com dispositivos eletrônicos associados ao ambiente real, sem desconectá-lo do espaço físico no qual se encontra (KIRNER; TORI, 2006). Estes autores descrevem que na RA, também compreendida como realidade mista (Mixed Reality), o real e o virtual interagem de maneira colaborativa ocorrendo sobreposição destes ambientes em tempo real, possibilitando grande variedade de experiências.

Em museus, a RA pode ser utilizada para expandir a experiência do visitante ou oferecer informações adicionais que não estejam disponíveis materialmente. Exemplos deste tipo de recurso, utilizados atualmente em instituições museológicas, são os aplicativos de smartphones que possibilitam a sobreposição em tempo real de gráficos 3D às imagens captadas pelas câmeras dos dispositivos no local da interação, oferecendo informações complementares ao ambiente real e às peças.

A utilização de recursos de RA em ambientes museológicos requer a consideração das múltiplas dimensões relacionadas ao processo, tanto em termos de mediação em museus, como na aplicação prática de projetos com bases tecnológicas. Trata-se de atividade multidisciplinar que envolve 
particularidades da instituição, seus públicos, projeto expográfico, questões técnicas de execução e avaliação dos resultados.

O termo Realidade Aumentada (RA) foi proposto por Tom P. Caudell e David W. Mizell em 1992, no artigo "Augmented reality: an application of heads-up display technology to manual manufacturing processes" (LEMOS; QUEIROZ, 2012; VAN KREVELEN; POELMAN, 2010). Entretanto, o primeiro protótipo de um sistema de RA foi implementado ainda nos anos de 1960, pelo professor de computação gráfica Ivan Sutherland e seus alunos das Universidades de Harvard e Utah. Nos anos de 1970 e 1980, centros de pesquisa no U.S. Air Force's Armstrong Laboratory e da NASA Ames, o Instituto de Tecnologia de Massachusetts e a Universidade da Carolina do Norte deram sequência às pesquisas sobre o tema (VAN KREVELEN; POELMAN, 2010).

Em outra vertente, a Realidade Virtual (RV) surgiu como um avanço dos periféricos e tecnologias de interface digital, a qual utiliza dispositivos multisensoriais para simular ambientes imersivos (reais ou imaginários) com os quais o usuário pode interagir em tempo real. Em RV utilizam-se recursos que permitem ao usuário interagir com dispositivos eletrônicos por meio de aparatos tecnológicos, tais como luvas, roupas, óculos ou capacetes, que obedecem aos comandos do usuário (KIRNER; TORI, 2006).

Para Kirner e Tori (2006), esta denominada "interface avançada do usuário" pode simular sensações por meio do estímulo dos sentidos e dos sentimentos do interlocutor. A principal diferença entre RA e RV diz respeito ao fato de que em RV tem-se a imersão completa no ambiente digital, enquanto na RA ocorre a sobreposição dos dois ambientes, o real e o virtual. Os usuários imersos em Ambientes Virtuais não são capazes de ver o mundo real, algo que em sistemas que utilizam RA é possível (AZUMA, 1997). A RA permite enriquecer o ambiente real por meio de dispositivos digitais, sem desconectar o usuário do espaço físico no qual se encontra (KIRNER; TORI, 2006).

Nesse sentido, a RA também pode ser descrita como Realidade Misturada (Mixed Reality), termo utilizado por Milgram e Kishino em 1994 (MILGRAM; KISHINO, 1994). O real e o virtual interagem de maneira 
colaborativa, ocorrendo sobreposição destes ambientes em tempo real, o que possibilita uma grande variedade de experiências (SILVA, 2015).

Azuma et al. (2001) buscaram descrever RA de forma a não limitar seu conceito ao tipo de tecnologia utilizada. Para tanto, os autores fornecem três características principais, gerais aos sistemas de RA:

a) combina objetos reais e virtuais em um ambiente real;

b) funciona de forma interativa e em tempo real;

c) registra (alinha) objetos reais e virtuais uns com os outros.

Para Van Krevelen e Poelman (2010), a adoção de tecnologias de RA pode melhorar a percepção dos usuários e ajudá-los a ver, ouvir e sentir melhor os ambientes que os cercam. Azuma (1997) afirma que a RA complementa a experiência com o objeto físico. Este autor descreve ainda que a RA pode ser pensada como o "termo intermediário" entre a Realidade Virtual (completamente virtual ou sintética) e o ambiente real. A RA pode ser compreendida como uma variação dos Ambientes Virtuais, ou da Realidade Virtual (AZUMA, 1997).

Diante deste referencial teórico, são apresentados a seguir os procedimentos adotados na presente investigação.

\section{Métodos de busca utilizados}

O método adotado envolveu a busca simples aplicada ao Catálogo de Teses e Dissertações - CAPES $^{8}$ e a Biblioteca Digital Brasileira de Teses e Dissertações - BDTD ${ }^{9}$. Não foi feito recorte temporal nas buscas, que abrangeram todo o período com produção disponível.

Por meio de múltiplas buscas, as palavras-chaves foram combinadas de modo a priorizar os termos "museu" e "tecnologias digitais" combinados de acordo com a seguinte lógica: $1^{\mathrm{o}}$ no título; $2^{\circ}$ no assunto; $3^{\circ}$ no resumo. Quando o número de trabalhos recuperados foi muito baixo, buscou-se em "qualquer lugar" do texto. Tal flexibilização mostrou-se adequada pelo caráter 
interdisciplinar e pela abrangência dos temas e termos que se relacionam com a prática de RA em ambiente museológico; isto permitiu a identificação de pesquisas que, mesmo não tratando exatamente do recorte proposto neste trabalho, dialogam com os temas e subtemas necessários para o corpus teórico para futuros estudos. Tal decisão também considerou o baixo volume de títulos recuperados com abordagem tão específica.

As palavras-chave utilizadas foram: museu; museologia; "RA"; "realidade aumentada"; "realidade mista"; "realidade virtual"; TIC; tecnologia; digital; háptica; QR-Code; RFID; RDA; NFC (near field communication); Beacon; tagpoint; "Código de Barra"; aplicativos; App; mobile; smartphone; celular; "dispositivos móveis"; tablet; interação; interativo; gamificação; "experiência do usuário"; UX; IHC; usabilidade; multimídia, mídia; ubíqua; pervasiva; interface; híbrida; ciberespaço; cibercultura; cibernético e inovação.

A seleção dos trabalhos passou por quatro processos de refinamento. No primeiro, foram coletados todos os títulos que relacionavam o uso de tecnologia digital no contexto museológico, incluindo museus virtuais e os trabalhos que tratam do emprego de RA no âmbito informacional. Os trabalhos que relacionam o uso de tecnologias digitais como suporte a manifestações relacionadas a atividades culturais também foram coletados nessa primeira etapa. Ao abrir o leque para os temas que extrapolam a RA e os ambientes dos museus, buscou-se definir os limites teóricos e termos tratados neste tipo de pesquisa. Com isso, novas palavras-chave foram sendo incorporadas ao longo do processo, resultando na lista supracitada.

No segundo processo de refinamento, partiu-se do critério de seleção de pesquisas que versam sobre as tecnologias interativas dentro do ambiente físico do museu. A partir dos resultados deste segundo refinamento, ocorreu a leitura dos resumos, que caracterizou a terceira etapa de filtragem. A recuperação do arquivo das teses e dissertações caracteriza a quarta etapa de seleção, uma vez que alguns títulos não estavam disponíveis. O produto final deste processo de quatro etapas é apresentado no tópico "Resultados".

Para a extração dos dados da literatura, aplicou-se o Checklist 1, apresentado a seguir. Este processo ocorreu por meio de análise dos metadados e 
dos conteúdos das produções, e utilizou os dados disponibilizados nas bases e nos próprios trabalhos. Na ausência destes, buscou-se identificar as informações no corpo dos trabalhos.

Checklist 1 - Dados extraídos dos trabalhos coletados

Título | Autor | Orientador | Banca | Ano | Tipo | Instituição | Programa

Área de conhecimento| Assuntos/Palavras-chave.

Fonte: Os autores.

O portal BDTD disponibiliza o campo "Trabalhos Relacionados", que também foi considerado no momento da busca. O fluxograma 1 apresenta as etapas.

Fluxograma 1 - Etapas da Revisão de Literatura

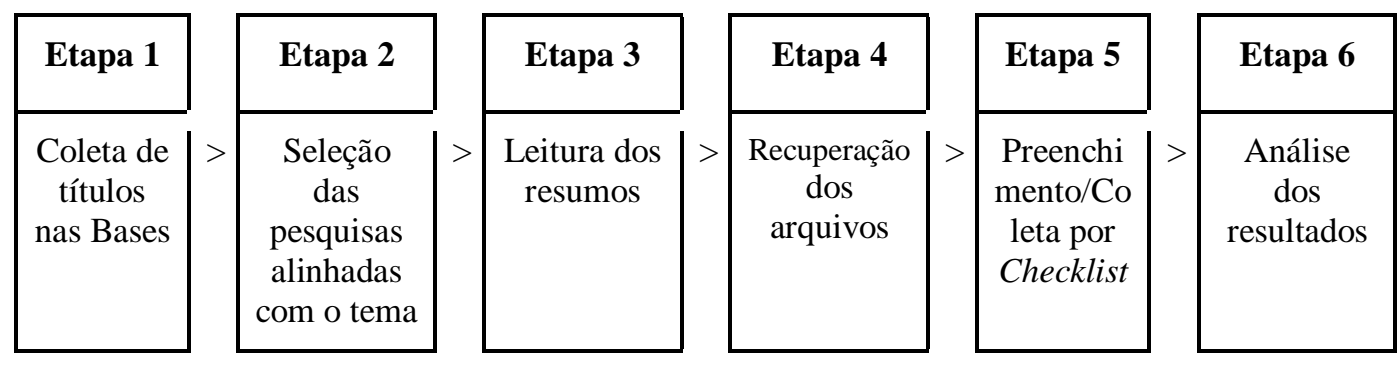

Fonte: Os autores.

As buscas ocorreram entre os meses de maio e julho do ano de 2017, sendo que em novembro de 2017 foi realizada nova consulta, esta com recorte de tempo relativo ao ano de 2017, para verificar possíveis atualizações. Todos os trabalhos pesquisados e coletados são de Programas de Pós-Graduação localizados no território brasileiro.

\section{Resultados}

No primeiro processo de refinamento - seleção dos títulos nas bases de dados foram obtidos 113 títulos no Banco de Teses e Dissertações CAPES e 48 da BDTD. Cabe ressaltar que a consulta ocorreu primeiro na Base CAPES, 
portanto, títulos repetidos foram naturalmente excluídos das buscas no BDTD. Em um processo de aferição, foram eliminados mais 7 trabalhos repetidos. $\mathrm{Na}$ segunda etapa - seleção de pesquisas que tratam do uso de tecnologias interativas dentro do ambiente físico do museu - o resultado foi de 38 trabalhos. Destas, 4 não estavam disponíveis na web e 17 não se encaixavam nos critérios estabelecidos pela presente proposta e, portanto, foram excluídos. Por fim, outros 17 trabalhos passaram por todas etapas do processo e compuseram o conjunto final de trabalhos a partir do qual foram extraídos os dados.

Dos 17 trabalhos deste conjunto, apenas $3(17,6 \%)$ utilizam o termo RA. Entretanto, foram encontradas as expressões "Realidade Mista", "Ambientes Híbridos", "Tecnologias Interativas", entre outros. Todos relacionados à concepção do que é RA. No que diz respeito à tipologia, $13(76,4 \%)$ são dissertações e $4(23,5 \%)$ são teses. Com relação aos anos de publicação, nota-se que o trabalho mais antigo é do ano de 2007 e os mais recentes, de 2017. O Gráfico 1 mostra que não há crescimento linear na quantidade de pesquisas ao longo dos anos, entretanto, entre os anos de 2011 e de 2017 concentra-se a maior produção sobre o assunto RA.

Gráfico 1 - Trabalhos publicados por ano

Trabalhos publicados sobre Tecnologias Interativas nos Museus (por ano).

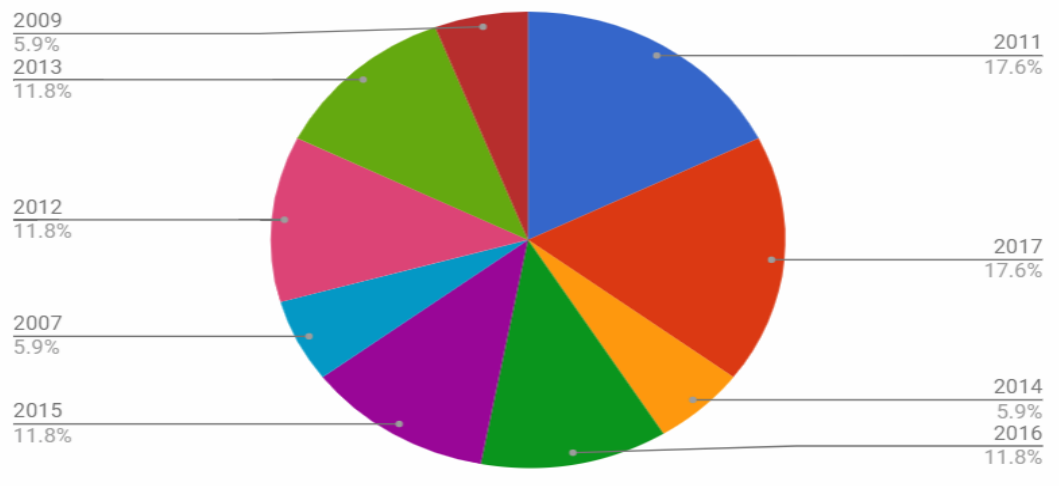

Fonte: Dados da pesquisa.

Neste conjunto de trabalhos também foram identificados $4(23,5 \%)$ projetos de natureza aplicada, ou seja, que apresentam algum tipo de produção de artefato tecnológico, e 9 (53\%) estudos de caso. Nestes últimos, o Museu da Língua Portuguesa (SP) e o Museu do Futebol (SP) são as instituições mais 
recorrentes. Quanto aos nomes de orientadores e membros de bancas, não houve repetições, revelando diversidade de atores, mesmo em um conjunto relativamente pequeno de trabalhos.

A Pontifícia Universidade Católica de São Paulo (PUC-SP) aparece vinculada a 3 trabalhos; a Universidade de São Paulo (USP) a 2; a Universidade Estadual Paulista (UNESP) a 2; a Universidade Federal do Rio de Janeiro (UFRJ) a 2; a Universidade Federal do Rio Grande do Sul (UFRGS) a 2; a Universidade Estadual de Campinas (UNICAMP) a 1; a Universidade Federal de Juiz de Fora (UFJF) a 1; a Universidade Federal de Minas Gerais (UFMG) a 1; a Universidade Federal de Santa Maria (UFSM) a 1; a Universidade Presbiteriana Mackenzie a 1 e a Universidade Regional de Blumenau (FURB) a 1. A Tabela 1 abaixo resume esta distribuição.

Tabela 1 - Quantidade de trabalhos por Instituição

\begin{tabular}{|l|c|}
\hline INSTITUIÇÃO & $\begin{array}{c}\text { QUANTIDADE DE } \\
\text { TRABALHOS }\end{array}$ \\
\hline PUC-SP & 3 \\
\hline USP & 2 \\
\hline UNESP & 2 \\
\hline UFRJ & 2 \\
\hline UFRGS & 1 \\
\hline UNICAMP & 1 \\
\hline UFJF & 1 \\
\hline UFMG & 1 \\
\hline UFSM & 1 \\
\hline Mackenzie & 1 \\
\hline FURB & \\
\hline
\end{tabular}

Fonte: Dados da pesquisa

Quanto às áreas de avaliação da CAPES, cabe salientar que estas foram alteradas em sua organização e algumas de suas denominações no ano de 2017. A área na qual se insere a CI, antes denominada como "Ciências Sociais Aplicadas", passou a ser identificada como "Comunicação e Informação". As 
áreas de Ciências Sociais Aplicadas > Arquitetura e Urbanismo e Ciências Sociais Aplicadas > Desenho Industrial passaram a receber a denominação Arquitetura, Urbanismo e Design. Para categorizar o trabalho associado à área de "Projeto, Espaço e Cultura", observou-se o programa ao qual está vinculado, sendo este a Faculdade de Arquitetura e Urbanismo da USP.

Sendo assim, foram utilizadas estas áreas atualizadas. Com isso, a área de "Comunicação e Informação" é a mais representada no conjunto pesquisado, com 5 trabalhos. Arquitetura, Urbanismo e Design aparece em 4 pesquisas (2 Arquitetura e Urbanismo, 1 Desenho Industrial e 1 Projeto, Espaço e Cultura). $\mathrm{Na}$ área da Educação, foram encontrados 3 trabalhos ${ }^{10}$, assim como na área Interdisciplinar. As áreas de engenharias (Engenharia Civil), Ciências Exatas e da Terra (Matemática) e Artes são representadas por 1 trabalho cada. A Tabela 2 ilustra estes dados.

Tabela 2 - Trabalhos por área de avaliação da CAPES

\begin{tabular}{|l|c|}
\hline \multicolumn{1}{|c|}{$\begin{array}{c}\text { ÁREA DE AVALIAÇÃO } \\
\text { DA CAPES }\end{array}$} & $\begin{array}{c}\text { QUANTIDADE DE } \\
\text { TRABALHOS }\end{array}$ \\
\hline Informação e Comunicação & 5 \\
\hline Arquitetura e Urbanismo & 3 \\
\hline Educação & 2 \\
\hline Interdisciplinar & 1 \\
\hline Artes & 1 \\
\hline Engenharias & 1 \\
\hline Exatas da Terra & 3 \\
\hline
\end{tabular}

Fonte: Dados da pesquisa.

No que se refere especificamente aos Programas de Pós-Graduação (PG), a maior ocorrência foi de apenas 2 trabalhos desenvolvidos em 2 programas: o Programa de Pós-Graduação em Comunicação e Semiótica da PUC-SP e o Programa de Pós-Graduação em Arquitetura e Urbanismo da USP. Todos os demais Programas de PG tiveram a ocorrência de apenas 1 trabalho em seu ambiente: Programa de PG em Arquitetura e Urbanismo da UFMG; 
Programa de PG em Artes Visuais da UFSM; Programas de PG em Comunicação e Informação da UFJF e da UFRGS; Programa de PG em Divulgação Científica e Cultural da UNICAMP; Programa de PG em Educação, Arte e História da Cultura da Mackenzie; Programa de PG em Educação da PUC-SP; Programa de PG em Engenharia da UFRJ; Programa de PG em Ensino de Ciências Naturais e Matemática da FURB; Programa de PG em Informática na Educação da UFRGS; Programa de PG em Mídia e Tecnologia da UNESP; Programa de PG em Museologia e Patrimônio da UNIRIO. O Programa de PG em CI da UNESP é o único nomeadamente inscrito no campo da CI. A Tabela 3 apresenta estes dados.

Tabela 3 - Quantidade de trabalhos por Programa de Pós-Graduação (PG)

\begin{tabular}{|c|c|}
\hline PROGRAMA DE PÓS-GRADUAÇÃO & $\begin{array}{l}\text { QUANTIDADE DE } \\
\text { TRABALHOS }\end{array}$ \\
\hline Programa de PG em Arquitetura e Urbanismo da USP & 2 \\
\hline Programa de PG em Comunicação e Semiótica da PUC-SP & 2 \\
\hline Programa de PG em Arquitetura e Urbanismo da UFMG & 1 \\
\hline Programa de PG em Artes Visuais da UFSM & 1 \\
\hline Programa de PG em Ciência da Informação da UNESP & 1 \\
\hline Programas de PG em Comunicação e Informação da UFJF & 1 \\
\hline Programa de PG em Comunicação e Informação da UFRGS & 1 \\
\hline $\begin{array}{c}\text { Programa de PG em Divulgação Científica e Cultural da } \\
\text { UNICAMP }\end{array}$ & 1 \\
\hline $\begin{array}{l}\text { Programa de PG em Educação, Arte e História da Cultura } \\
\text { da Mackenzie }\end{array}$ & 1 \\
\hline Programa de PG em Educação da PUC-SP & 1 \\
\hline Programa de PG em Engenharia da UFRJ & 1 \\
\hline $\begin{array}{c}\text { Programa de PG em Ensino de Ciências Naturais e } \\
\text { Matemática da FURB }\end{array}$ & 1 \\
\hline Programa de PG em Informática na Educação da UFRGS & 1 \\
\hline Programa de PG em Mídia e Tecnologia da UNESP & 1 \\
\hline Programa de PG em Museologia e Patrimônio da UFRJ & 1 \\
\hline
\end{tabular}

Fonte: Dados da pesquisa. 
Vale ponderar que, como o foco da busca se concentrou nas ocorrências de estudos sobre RA em museus e/ou centros culturais (ambientes, por natureza, baseados em informação), estes resultados não espelham os estudos relativos à RA em geral. Trata-se da aplicação de um recurso de cunho tecnológico nestas organizações, e consequentemente, os trabalhos foram produzidos em maior quantidade por Programas de Pós-Graduação de áreas mais associadas às Ciências Sociais Aplicadas, como Comunicação e Informação, Arquitetura e Urbanismo, Museologia, Ciência da Informação (com 9 trabalhos distribuídos em 7 programas), além de Educação, Artes e Interdisciplinar (com 6 trabalhos distribuídos em 6 programas); do que as áreas de Engenharias e Exatas (com 2 trabalhos distribuídos em 2 programas).

\section{Considerações finais}

O presente trabalho objetivou identificar os estudos acadêmicos na área da CI no que tange à questão da inserção de mídias digitais interativas, em especial a RA, em espaços museológicos. Para tanto, o método estipulado de busca envolveu o exame das bases de teses e dissertações dos Programas de Pós-Graduação no Brasil. Constatou-se que a representatividade da produção em CI nestes temas ainda é baixa em relação à produção total sobre os mesmos, indicando oportunidades para o desenvolvimento de estudos aplicados com base nos fundamentos teóricos e práticos da área de CI, o que seria uma contribuição desejável em termos interdisciplinares.

O fato de não haver nomes de destaque, no que diz respeito à frequência de participação, dentre orientadores e membros das bancas nos trabalhos que tratam sobre estes temas em conjunto assinala heterogeneidade e dispersão com relação aos investigadores do tema, e pode ser um indício de que não há especialistas com pesquisas sistemáticas e continuadas nesta temática no Brasil.

No mesmo sentido, a distribuição dos trabalhos por diversos Programas de Pós-Graduação demonstra que as pesquisas sobre o tema ainda estão em estágios iniciais e/ou incipientes, ou que ainda não estão consolidadas em 
grupos de pesquisa. Tal resultado aponta para novas possibilidades na adoção de uma postura interdisciplinar, quiçá a criação de um protocolo de pesquisa na medida em que o aporte conceitual e tecnológico de outras ciências possibilita novos diálogos e interações, ampliando assim a capacidade de ação da CI.

A CI é uma área de conhecimento relativamente nova que vem, ao longo dos anos, aparando suas arestas a fim de sustentar um campo sólido de conhecimento, com pesquisas e práticas que assumam a identidade dessa "jovem ciência" de maneira integrada a partir de novas realidades, conforme apresentado na seção 2 .

Portanto, compreende-se que ainda há vasto domínio a ser explorado e lacunas a serem preenchidas sob a perspectiva da CI, no que tange ao estudo de RA em contextos museológicos e informacionais, e que estes temas demandam e comportam maiores aprofundamentos.

\section{Referências}

ALMEIDA, Maria Christina Barbosa. Revista Conhecimento em Ação, Rio de Janeiro, v. 1, n. 1, jan./jun. 2016.

APLICATIVO serve como guia para visitantes da exposição de Gaudí.Diário Catarinense, Florianópolis, 01 set. 2016.

ARAÚJO, Carlos Alberto Ávila. Arquivologia, Biblioteconomia, Museologia e CI: o diálogo possível. São Paulo: Briquet de Lemos, 2014.

AZUMA, Ronald T. A Survey of Augmented Reality. Presence: teleoperators and virtual environments, Cambridge, v. 6, n. 4, p. 355-385, 1997.

AZUMA, Ronald et al. Recent Advances in Augmented Reality. IEEE Computer Graphics and Applications, Piscataway, v. 21, n. 6, 2001.

CAUDELL, Tom; MIZELI, David. Augmented reality: an application of headsup display technology to manual manufacturing processes. In:

INTERNATIONAL CONFERENCE SYSTEM SCIENCES, 25., 1992, Hawaii. Proceedings[...] Hawaii: IEEE,1992. p. 659-669.

CARVALHO, R. M. R de. Comunicação e informação de museus na Internet e o visitante virtual. Museologia e Patrimônio, Rio de Janeiro, v. 1, n. 1, p. 8393, jul./dez. 2008. 
CHELINI, Maria Júlia Estefânia. Novas tecnologias para... novas (?) expografias. Museologia \& Interdisciplinaridade, Brasília, v. 1, n. 2, p. 59-71, 2012.

CURY, Marília Xavier. Comunicação Museológica: Uma Perspectiva Teórica e Metodológica de Recepção. 2005. 366 F. Tese (Doutorado) - Curso de Ciências da Comunicação, Escola de Comunicações e Artes da Universidade de São Paulo, São Paulo, 2005.

DESVAllÉES A.; MAIRESSE, F. Conceitos-chave de Museologia. Comitê Brasileiro do Conselho Internacional de Museus: São Paulo, 2013.

HE, Zhiqiang; CUI, Binyue; ZHOU, Wei. A proposal of interaction system between visitor and collection in museum hall by iBeacon. In:

INTERNATIONAL CONFERENCE ON COMPUTER SCIENCE \& EDUCATION (ICCSE), 10., 2015, Cambridge. Proceedings[...] Cambridge: IEEE, 2015. v. 10, p. 427-430.

HOMULUS, Peter. Museums to libraries: a family of collecting institutions. Art Libraries Journal, Cambridge, v.15, n.1, p.11-13, 1990.

KIRNER, Claudio; TORI, Romero. Fundamentos da RA. In: KIRNER, Claudio; TORI, Romero; SISCOUTO, Robson (Orgs.). Fundamentos e Tecnologia da Realidade Virtual e Aumentada. Belém: SVR, 2006. p. 22-38.

LEMOS, André; QUEIROZ, Camila. Memórias soteropolitanas: RA na cidade do Salvador. Inclusão Social, Brasília, DF, v. 5, n. 2, p.128-136, jun. 2012.

LEMOS, Joana Gusmão. Perspectivas transdisciplinares de aproximação com a Ciência sob o olhar da CI: uma metodologia bottom-up para a TV Unesp. 2016. Tese (Doutorado em CI) - Universidade Estadual Paulista "Júlio de Mesquita Filho", Faculdade de Filosofia e Ciências, Campus de Marília/SP, 2016.

LIU, Yue; YANG, Ju; LIU, Mingjun. Recognition of QR Code with mobile phones. In: CHINESE CONTROL AND DECISION CONFERENCE, Yantai, 2008, Proceedings [...] Yantai: IEE, p.203-206, Jul. 2008.

MILGRAM, Paul, KISHINO, Fumio. A taxonomy of mixed reality visual displays. IEICE Transactions on Information Systems, [s.l.], v. E77-D, n. 12, Dec. 1994.

MUCHACHO, Rute. Museus virtuais: A importância da usabilidade na mediação entre o público e o objecto museológico. In: CONGRESSO DA ASSOCIAÇÃO PORTUGUESA DE CIÊNCIAS DA COMUNICAÇÃO, 4., 2005, Aveiro, Anais [...] Aveiro: SOPCOM, 2005. p. 1540-1547.

MUSEUM Definition. Vienna: ICOM, 2007. 
PINHO, Joana Maria Balsa Carvalho de. Museus e Internet. Recursos online nos sítios web dos museus nacionais portugueses. Revista Textos de la

CiberSociedad, La Rioja, n. 8, 2007.

PRASS, Ronaldo. Entenda o que são os 'QR Codes', códigos lidos pelos celulares. Rio de Janeiro: Globo, 2011.

PRENSKY, M. Nativos digitais, imigrantes digitais. Califórnia: NBC University Press, 2001.

REIS, Raphael; BARBOSA, Valéria. Museus de Sergipe: RA e documentação na Museologia. In: ENCONTRO REGIONAL DE HISTÓRIA DA ANPUHRIO: Saberes e práticas científicas, 16., 2014, Rio de Janeiro. Anais[...] Rio de Janeiro: ANPUH, 2014.

SMIT, Johanna W. Arquivologia, CI e as duas faces de Jano. Brazilian Journal of Information Science: Research Trends, Marília, v. 11, n. 4, p. 6-9, 2017.

SMIT, Johanna W. O documento audiovisual ou a proximidade entre as 3 Marias. Revista Brasileira de Biblioteconomia e Documentação, São Paulo, v. 26, n. 1/2, p. 81-85, 1993.

SARACEVIC, Tefko. CI: origem, evolução e relações. Perspectivas em CI, Belo Horizonte, v.1, n. 1, p. 41-62, jan./jun., 1996.

SHERA, Jesse H., CLEVELAND, Donald B. History and foundations of Information Science. ARIST: annual review of information science and technology, Hoboken, v. 12, p. 249-275, 1977.

SILVA, Carlos Guilherme Marques Nunes da. O potencial da RA no turismo: o caso dos museus. 2015. 96 f. Dissertação (Mestrado em Gestão) Universidade Católica Portuguesa, Porto, 2015.

SOUSA, Antônio Augusto de. Espaços Museológicos Virtuais a Villa Romana do Rabaçal: estudo de caso. Tese (Doutorado) - Universidade do Porto, Porto, 2007.

VAN KREVELEN, D.W.F.; POELMAN, R. A Survey of Augmented Reality Technologies, Applications and Limitations. The International Journal of Virtual Reality, Brest, v. 2, n. 9, p. 1-20, jan. 2010.

VELOSO, Gabrielli Ciasca. Avaliação da Interface de Interação para a Aplicação Multimídia do Totem Digital do Museu Histórico de Araranguá. 2015. 138 f. TCC (Graduação) - Curso de Tecnologias da Informação e Comunicação, Universidade Federal de Santa Catarina, Araranguá, 2015.

ZAGATTO, Vanessa Alves. Tecnologias de informação e comunicação e o ambiente museológico: um estudo do discurso tecnológico dos periódicos. 
2013. Dissertação (mestrado) - Universidade Estadual Paulista, Faculdade de Filosofia e Ciências de Marília, 2013.

\title{
The use of Augmented Reality in the context of museums: the Brazilian portfolio of theses and dissertations until 2017
}

\begin{abstract}
The objective of this study is to identify and analyze within the literature from Brazilian Postgraduate programs theses and dissertations that investigated the use of digital technologies, namely Augmented Reality (AR) in museum spaces. It is justified in view of the development of the theme based on consolidated studies in Brazilian postgraduate programs and the identification of theoretical basis for future research. For this purpose, a simple, preliminary and exploratory search was used in the Coordenação de Aperfeiçoamento de Pessoal de Nível Superior and in the Instituto Brasileiro de Informação em Ciência e Tecnologia Thesis and Dissertation Databases. The results pointed to a dispersed and little systematized production and the opportunity to create research protocols on the subject based on the data collected.
\end{abstract}

Keywords: Museum. Augmented Reality. Information Science. Interdisciplinarity. Museology.

Recebido: 22/08/2018

Aceito: $14 / 11 / 2018$

1 MUSEU DA LÍNGUA PORTGUESA. São Paulo, 2019. Disponível em: http://www.museudalinguaportuguesa.org.br. Acesso em: 20 mai. 2019.

${ }^{2}$ Antes do incêndio ocorrido em 21 de dezembro de 2015.

3 VISITA virtual. São Paulo: Museu da casa portinari, 2019. Disponível em: https://www.museucasadeportinari.org.br/visite-o-museu/visita-virtual-2. Acesso em: 20 mai. 2019.

4 GOOGLE. Google arts \& culture. Mountain View: Google, c2015. Disponível em: https://www.google.com/culturalinstitute/beta/. Acesso em: 20 mai. 2019

${ }^{5}$ Código de Resposta Rápida.

${ }^{6}$ NOVAS TECNOLOGIAS | QR CODE no Museu. Nazaré: Museu Dr. Joaquim Manso, 2016. Disponível em: http://mdjm-nazare.blogspot.com.br/2016/06/novas-tecnologias-na-exposicaoqr-code.html. Acesso em: 20 mai. 2019.

7 MUSEU Dr. Joaquim Manso. Nazaré: Câmara Municipal da Nazaré, 2019. Disponível em: http://www.cm-nazare.pt/pt/museu-dr-joaquim-manso. Acesso em: 20 mai. 2019

${ }^{8}$ Coordenação de Aperfeiçoamento de Pessoal de Nível Superior - CAPES. Catálogo de Teses e Dissertações. c2016. Disponível em: http://catalogodeteses.capes.gov.br/catalogo-teses/. Acesso em: 20 mai. 2019

${ }^{9}$ Biblioteca Digital Brasileira de Teses e dissertações - BDTD. Acesso e visibilidade às teses e dissertações brasileiras. Brasília: IBICT, 2019 Disponível em: http://bdtd.ibict.br/vufind/. Acesso em: 20 mai. 2019

${ }^{10} \mathrm{O}$ trabalho do Programa de Pós-Graduação em Educação, Arte e História da Cultura foi inserido dentro da área da Educação, devido a natureza de seu conteúdo. 